\title{
TECNOLOGIA MÓVEL À BEIRA DO LEITO: PROCESSO DE ENFERMAGEM INFORMATIZADO EM TERAPIA INTENSIVA A PARTIR DA CIPE 1.0 ${ }^{\circledR}$
}

\author{
Daniela Couto Carvalho Barra1, Grace Teresinha Marcon Dal Sasso ${ }^{2}$
}

\footnotetext{
${ }^{1}$ Doutoranda pelo Programa de Pós-Graduação em Enfermagem (PEN) da Universidade Federal de Santa Catarina (UFSC). Bolsista CNPq. E-mail: danyccbarra@yahoo.com.br

${ }^{2}$ Doutora em Enfermagem. Docente do Departamento de Enfermagem e do PEN/UFSC. Santa Catarina, Brasil. E-mail: grace@ ccs.ufsc.br
}

RESUMO: Produção tecnológica e estudo metodológico baseado na evidência que avaliou com os enfermeiros de Terapia Intensiva os critérios de Ergonomia e Usabilidade do Processo de Enfermagem Informatizado desenvolvido em um dispositivo tecnológico móvel a partir da Classificação Internacional para as Práticas de Enfermagem versão 1.0. O estudo foi conduzido de maio a junho de 2008 com 13 avaliadores. Os dados foram analisados mediante o estabelecimento de médias e refletidos a partir da Classificação Internacional para as Práticas de Enfermagem versão 1.0 e das evidências dos estudos na área. O estudo mostra que o sistema possui critérios de Ergonomia e Usabilidade de acordo com a Organização Internacional de Padrões de Sistemas. Sobretudo, o sistema desenvolvido tem aplicação prática porque permite avaliar, intervir e gerenciar o cuidado de enfermagem proporcionando maior segurança, conhecimento e envolvimento dos enfermeiros com as necessidades do paciente à beira do leito.

DESCRITORES: Processos de enfermagem. Unidades de terapia intensiva. Informática em enfermagem. Tecnologia da informação. Terminologia.

\section{MOBILE BEDSIDE TECHNOLOGY: COMPUTERIZED NURSING PROCESSES IN INTENSIVE CARE UNIT FROM ICNP 1.0}

\begin{abstract}
This technological production and methodological evidence-based study aimed to evaluate with Intensive Care Unit nurses the criteria of Ergonomics and Usability of the computerized Nursing Process in a mobile device from the International Classification for Nursing Practices version 1.0. The study was conducted from May to June of 2008, with 13 appraisers. The data was analyzed by establishing averages and reflected based on the International Classification for Nursing Practices and the evidence of studies in the area. This study shows that the system has Ergonomics and Usability criteria according to the International Systems Standards Organization. Above all, the system developed has practical applications because it enables decision makers to assess, intervene, and manage nursing care providing greater security, knowledge, and involvement of nurses with the bedside needs of the patient.
\end{abstract}

DESCRIPTORS: Nursing process. Intensive care units. Nursing informatics. Information technology. Terminology.

\section{TECNOLOGÍA MÓVIL EN LA CABECERA DEL LECHO: UN PROCESO INFORMATIZADO DE ENFERMERÍA EN UNIDADES DE CUIDADOS INTENSIVOS A PARTIR DE LA CIPE 1.0 ${ }^{\circledR}$}

RESUMEN: Producción tecnológica e investigación metodológica basada en la evidencia que tuvo por objeto evaluar, con los enfermeros de las Unidades de Cuidados Intensivos, los criterios de Ergonomía y Usabilidad de los sistemas computadorizados del Proceso de Enfermería desarrollado en un dispositivo tecnológico móvil, a partir de la Clasificación Internacional para las Prácticas de Enfermería versión 1.0. El estudio se realizó de mayo a junio de 2008 con 13 evaluadores. Los datos se analizaron mediante el establecimiento de promedios y fueron considerados a partir de la Clasificación Internacional para las Prácticas de Enfermería y de las evidencias de los estudios en el área. La investigación muestra que el sistema tiene criterios de Ergonomía y Usabilidad que están de acuerdo con la Organización Internacional de Patrones de Sistemas. El sistema desarrollado tiene aplicación práctica ya que permite evaluar, intervenir y gestionar la prestación de cuidados de enfermería proporcionando una mayor seguridad, el conocimiento y la participación de los enfermeros con las necesidades del paciente en la cabecera del lecho.

DESCRIPTORES: Procesos de enfermería. Unidades de terapia intensiva. Informática aplicada a la enfermería. Tecnología de la información. Terminología. 


\section{INTRODUÇÃO}

Numa perspectiva atual, a tecnologia tem se manifestado de modo crescente dentro de um sistema tecnológico nos quais os governos, as organizações e as pessoas estão integradas com o objetivo de maximizar a eficiência e a racionalidade. É, portanto, um erro supor que a inovação tecnológica tem apenas um efeito unilateral e que, igualmente, é um erro definir a tecnologia apenas como instrumentos e técnicas ou associá-la a compreensão de superioridade, especialização e profissionalismo. A tecnologia influência, portanto, o mundo em torno das pessoas, neste estudo, o ambiente da prática de enfermagem em terapia intensiva. ${ }^{1}$

O cuidado de enfermagem, especialmente em Unidades de Terapia Intensiva (UTI), é complexo e desafiador. Os enfermeiros estão regularmente expostos a situações clínicas difíceis que requerem atenção e controle e, ao mesmo tempo, a inovações tecnológicas que precisam estar integradas de forma consistente, correta e segura ao sistema de cuidado à beira do leito. Cada tecnologia adicionada ao cuidado e ao sistema organizacional aumenta a complexidade do trabalho da enfermagem embora, também, potencialize a redução da carga de trabalho, melhorando a qualidade do cuidado e diminuindo os erros e eventos adversos. ${ }^{2}$

Dentre as diversas tecnologias utilizadas e/ou desenvolvidas na prática, o Processo de Enfermagem (PE) melhora a qualidade do cuidado por permitir ao enfermeiro sistematizar suas intervenções de forma clara e organizada, centrada nas necessidades dos clientes. O PE é, portanto, uma tecnologia de cuidado que orienta uma sequência de raciocínio lógico, constituindose numa ferramenta que pode ser utilizada pelos enfermeiros na prática profissional, evidenciando o desencadeamento dos pensamentos e juízos desenvolvidos durante a assistência. ${ }^{1-6}$

Em um setor como a UTI, o PE além de integrar, organizar e garantir a continuidade das informações da equipe de enfermagem permite avaliar a sua eficácia e efetividade e, modificá-lo de acordo com os resultados na recuperação do cliente e também servir de fundamentação permanente para a educação, pesquisa e gerenciamento em enfermagem.

Não há dúvidas que o volume de informações na UTI, assim como nos demais cenários de cuidado é enorme e que estas informações são heterogêneas, distribuídas, complexas e pouco estruturadas. Informações são centrais no processo de cuidar em saúde. Ou seja, o acesso à informação sobre a prática, fortalece os enfermeiros com evidências para sustentar as contribuições da enfermagem nos resultados do cliente. Portanto, existe uma conexão essencial entre o acesso à informação pelo PE, os resultados e a segurança do cliente. ${ }^{7}$

Neste contexto, as Tecnologias da Informação e Comunicação (TICs) têm sido utilizadas como um caminho para apoiar o desenvolvimento do PE, pois permite integrá-lo em uma estrutura lógica de dados, informação e conhecimento para a tomada de decisão do cuidado de enfermagem. ${ }^{8}$ Assim, para integrar estas informações do $\mathrm{PE}$, diversas terminologias de enfermagem têm sido desenvolvidas e estudadas nos últimos anos e a Classificação Internacional para as Práticas de Enfermagem $\left(\mathrm{CIPE}^{\circledR}\right)$, na sua versão 1.0, estruturada para ser informatizada, é um modelo de sete eixos que estabelece os diagnósticos de enfermagem, os resultados e as intervenções do enfermeiro de acordo com as necessidades prioritárias de saúde do cliente. ${ }^{9-10}$

Considera-se, portanto, que a integração entre o PE e as TICs pode propiciar a melhoria do pensamento crítico; a aproximação desses profissionais com os cuidados intensivos; a discussão clínica entre os pares e equipe multidisciplinar; o desenvolvimento do raciocínio investigativo e; o fomento da busca contínua de informações que visam obter evidência científica. Vários estudos corroboram com esta perspectiva ao considerar que as TICs melhoram o cuidado direto, os resultados do cliente e os ambientes da prática, bem como, reduzem o tempo de documentação e registro. ${ }^{1-2,11-13}$

Sob outro ângulo, aspectos negativos das TICs e das demais tecnologias dos ambientes de cuidado têm sido identificados especialmente relacionados com o uso, o reparo e a manutenção de equipamentos; falta de oportunidades de aprendizagem para as novas tecnologias, bem como, tecnologias ergonomicamente inapropriadas (pesadas, distantes do leito, design inadequado, etc.). ${ }^{2}$

Neste contexto, visando aproximar o enfermeiro do cuidado ao cliente em terapia intensiva e contribuir para a sua segurança, controle e o gerenciamento da prática de enfermagem a partir da utilização de uma tecnologia informatizada, este estudo descreve a avaliação dos critérios de Ergonomia e Usabilidade do PE informatizado em dispositivo tecnológico móvel tipo Personal Digital Assistant (PDA) a partir da CIPE ${ }^{\circledR} 1.0$. 
A descrição desta avaliação é uma parte do estudo de Dissertação de Mestrado intitulado - Processo de Enfermagem informatizado em terapia intensiva em ambiente PDA a partir da $\mathrm{CIPE}^{\circledR}$ versão $1.0 .{ }^{14}$ Torna-se necessário salientar que este estudo é uma continuidade do sistema informatizado para PE em UTI que vem sendo desenvolvido em estudos de 1999, 2002 e 2006 para ambiente Web. ${ }^{15-17}$

\section{MÉTODO}

Trata-se de uma produção tecnológica e estudo metodológico ${ }^{18}$ baseado na evidência, ${ }^{3}$ que avaliou com os enfermeiros da UTI a Ergonomia e a Usabilidade do PE informatizado no cuidado de enfermagem a beira do leito. A ergonomia compreende as categorias organização, interface, técnico e conteúdo do sistema. O conteúdo, a partir da estruturação dos dados, é a capacidade do sistema atender adequadamente as necessidades do cliente e estabelecer a assistência mais eficaz visando sua recuperação. A usabilidade mede a eficácia, a eficiência e a satisfação com que um usuário pode realizar um conjunto específico de tarefas em um ambiente particular; dentre seus objetivos destacam-se o de avaliar se tarefas simples e básicas são fáceis de serem desempenhadas pelos usuários. Ou seja, um sistema é considerado eficaz quando permite que os usuários atinjam seus objetivos. ${ }^{19}$

O estudo foi conduzido no período de maio a junho de 2008 em duas UTIs gerais de dois hospitais de grande porte do estado de Santa Catarina - Brasil.

A população foi constituída por uma amostra intencional não probabilística por julgamento, composta por 13 avaliadores sendo: nove enfermeiros, dois educadores da área de terapia intensiva e dois programadores de sistemas. Os critérios para inclusão no estudo foram: aceitar por livre e espontânea vontade participar do estudo mediante assinatura do Termo de Consentimento Livre e Esclarecido (TCLE); ser enfermeiro da UTI para a avaliação do Processo de Enfermagem informatizado; ser enfermeiro docente ou pesquisador na área da terapia intensiva; ser programador de sistemas formado ou no último ano de formação (específico para a avaliação do sistema informatizado) para avaliação de acordo com a International Standards Organization e International Electrotechnical Commission 9241-11 (ISO/IEC), ${ }^{19}$ que estabelece os critérios de Ergonomia e Usabilidade de um sistema de informação.
Para a realização deste estudo foram respeitados os preceitos éticos determinados pela Resolução N¹96/96 do Conselho Nacional de Saúde mediante o cumprimento das exigências do TCLE, especialmente no direito à informação e a liberdade para desistir do estudo a qualquer momento. ${ }^{20}$ Por se tratar de um estudo que envolveu a participação de seres humanos foram também adotados os procedimentos explicitados na Declaração de Helsinki e os cinco referencias básicos da Bioética, quais sejam: autonomia, nãomaleficência, beneficência, justiça e anonimato. Neste sentido, os participantes do estudo foram identificados pelas seguintes siglas: Enfermeiro (E1, E2, e assim sucessivamente); Professor (Pf1 e Pf2) e; Programadores de Sistemas (Pg1 e Pg2).

Este estudo foi submetido ao Comitê de Ética em Pesquisa da Universidade Federal de Santa Catarina e ao Comitê de Ética em Pesquisa do Hospital Regional Homero de Miranda Gomes, da Secretaria Estadual de Saúde de Santa Catarina sendo aprovado sem ressalvas, pelos membros das duas entidades.

O instrumento para a coleta de dados constituiu-se de um questionário fundamentado na ISO $9241-11^{19}$ e mediu os critérios de Ergonomia e Usabilidade do sistema informatizado em dispositivo móvel para o PE em Terapia Intensiva, a partir da $\mathrm{CIPE}^{\circledR}$ versão 1.0. No critério Ergonomia quatro categorias foram avaliadas: organização (três itens) interface (quatro itens), conteúdo (dois itens) e técnico (três itens). No critério Usabilidade do PE informatizado 19 itens foram avaliados pelos enfermeiros, professores e programadores de sistemas. Ressalta-se que neste instrumento de avaliação havia questões abertas para que os participantes complementassem suas avaliações.

Neste estudo, optou-se por excluir os programadores de sistemas da avaliação da categoria Conteúdo, uma vez que os mesmos não são enfermeiros e, portanto, desconhecem se as informações/conteúdo são adequadas e suficientes e se atendem as expectativas dos enfermeiros para implementação do PE informatizado.

Os itens no instrumento de avaliação foram distribuídos em uma escala de valores com as seguintes categorias de respostas: 5- Excelente (E), 4- Muito Bom (MB), 3- Bom (B), 2- Regular (RE), 1- Ruim (R). Considerou-se na avaliação que os valores da média entre: 1 a 1,5 receberiam a classificação (RUIM); de 1,51 a 2,5 (REGULAR); de 2,51 a 3,5 (BOM); de 3,51 a 4,5 (MUITO BOM) e de 4,51 a 5 (EXCELENTE). 
Os dados foram analisados mediante estatística descritiva com cálculos de média dos critérios de ergonomia e usabilidade e os relatos dos enfermeiros foram discutidos a partir do referencial da $\mathrm{CIPE}^{\circledR}$ versão 1.0 e das evidências dos estudos de referência na área. Ou seja, para cada conjunto de critérios de avaliação identificados pelos enfermeiros, buscou-se sustentar e ou contestar a evidência.

\section{RESULTADOS E DISCUSSÃO}

\section{Avaliação do critério ergonomia}

Observou-se durante a coleta de dados que a categoria Organização do critério de Ergonomia obteve média geral 4,38, considerada $\mathrm{MB}$ pelos avaliadores, demonstrando que a organização e o modo de acesso aos arquivos favorecem a uma execução eficiente do sistema (média 4,46); que o sistema é de fácil operacionalização (média 4,08) e que o sistema atinge os objetivos para a assistência de enfermagem em UTI utilizando a $\mathrm{CIPE}^{\circledR}$ (média 4,62).

No relato dos avaliadores foi possível verificar que o sistema possui: Organização, acesso fácil às informações que são claras e coerentes. Facilidade em acessar as informações e alterá-las se necessário (E8).

Fácil navegação, informações claras, etapas sucintas (E9); Fácil acesso, flexibilidade, portabilidade e facilidade de uso (Pf1).

O sistema é de fácil uso e atende a necessidade de avaliação do paciente em estado grave, dando suporte e agilidade ao enfermeiro para a realização desta atividade [o processo de enfermagem] (Pf2).

A mudança do processo de registro de dados e informações em papel para o registro eletrônico impõe desafios aos profissionais de saúde. Contudo, estudos apontam que o principal esforço da Informática em Enfermagem é especificar as exigências para o sistema de informação clínica e incluir as necessidades dos enfermeiros no processamento das informações para apoiar a prática. Em um sistema informacional é preciso integrar pessoas, informação, procedimentos, recursos de computação em um objetivo comum de maximizar os benefícios aos seres humanos e as capacidades tecnológicas. Para estes benefícios realmente acontecerem na prática, torna-se imperativo que os fluxos de trabalho e as rotinas sejam revisados, repensados ou alterados. ${ }^{8,21-25}$

Estudos ainda apontam que a utilização do registro eletrônico por enfermeiros e médicos está diretamente relacionada à sua satisfação com os sistemas $^{26-28}$ e que a satisfação quanto ao uso de registro eletrônico nos ambientes de saúde varia de baixa a alta. ${ }^{27,29-31}$

$\mathrm{Na}$ categoria Interface a média geral foi considerada MB $(4,35)$ pelos avaliadores destacando: aparência das telas (média 4,08); estrutura lógica dos dados - as informações aparecem ao usuário logicamente estruturadas (média 4,69); a quantidade de informação é suficiente para a assistência de enfermagem em terapia intensiva - coleta de dados, diagnósticos e intervenções (média 4,69) e; conforto visual para manuseio do sistema (média 3,92).

Percebe-se, nos relatos dos avaliadores em relação aos itens avaliados do critério Interface, que a estruturação lógica dos dados e a quantidade de informações estão muito completas e por vezes, extensas: Facilidade para fazer o diagnóstico e acho que quando a pessoa adquirir a prática será mais fácil e ganhará tempo (E2).

Por ser muito completo ele exige muito tempo do enfermeiro, porém, com certeza, com o uso frequente, este tempo diminuiria (E3).

A princípio, pode-se gerar uma prescrição muito extensa; rever objetividade. Acredito que temos realmente muitos cuidados a serem prestados, porém sua extensão pode gerar desinteresse pela falta de objetividade (E9).

Tal constatação dos enfermeiros também foi observada em estudos que examinaram a qualidade da documentação do registro médico eletrônico e detectaram que o potencial para o registro de informação inadequada, a dificuldade de acesso e a recuperação das informações dos pacientes são as principais causas de preocupação dos profissionais. Os estudos recomendam que maior atenção deve estar focalizada no design da interface do usuário e na customização do ambiente do fluxo de trabalho dos profissionais de saúde, ao qual melhoraria a satisfação do usuário e a confiança na exatidão das informações, bem como, diminuiria a sobrecarga de trabalho cognitiva dos profissionais. ${ }^{4,13,32}$

Quanto à questão do tempo relatado pelos enfermeiros, estudos também revelam que o registro eletrônico afeta a eficiência do tempo na prática, incluindo especialmente o tempo para a documentação. ${ }^{29-30,33-34}$ Por outro lado, outro estudo afirma que o registro eletrônico reduz o tempo de documentação dos enfermeiros na prática assistencial. ${ }^{2}$

Neste sentido, o sucesso ou o fracasso de um sistema de informação clínica na prática está relacionado com o seu acesso à cultura do ambiente de trabalho, às práticas de trabalho, $\mathrm{e}$ 
as necessidades de informação dos usuários. Sistemas que não levam em consideração benefícios importantes aos usuários e o contex to de trabalho dos profissionais podem levar a resistências especialmente na recusa no uso do sistema, críticas ou minimização de seu uso. ${ }^{35}$

Neste estudo, optou-se por construir um sistema que contemplasse de forma mais completa possível o processo de avaliação clínica do cliente UTI para proporcionar aos enfermeiros maior segurança na tomada de decisão da assistência de enfermagem de acordo com a complexidade das situações clínicas que envolvem os clientes de terapia intensiva. Contudo, novos estudos precisam ser conduzidos para diminuir a sobrecarga de trabalho dos enfermeiros e melhorar a integração com seus fluxos de trabalho.

Quanto ao conforto visual para manuseio do sistema, os participantes atribuíram menor média $(3,92)$. Tal fato pode estar relacionado a diversos fatores tais como: tipo de dispositivo móvel utilizado (TREO 650) ainda pouco utilizado pelos profissionais de enfermagem; tamanho reduzido da tela do PDA; as características específicas dos avaliadores; sistema informatizado ser novo e pouco conhecido pelos avaliadores e a pouca habilidade em manusear o dispositivo.

As falas a seguir explicitam algumas dificuldades encontradas pelos avaliadores durante a avaliação da categoria Interface do sistema informatizado referente à aparência das telas e conforto visual para manuseio do sistema: dificuldade de operacionalização do palmtop; telas [interface] pequenas e letras miúdas e condensadas; combos de difícil visualização para relacionar ao conteúdo (E6).

As telas do sistema são claras e utilizam cores adequadas, mas as letras poderiam ser maiores para facilitar a leitura e interpretação, bem como o elenco dos diagnósticos e das intervenções poderiam seguir a ordem alfabética para facilitar a identificação (Pf1).

Estudos mostram que a utilização de dispositivos móveis à beira do leito para o registro eletrônico de saúde dos clientes será cada vez mais disponibilizada nos diversos ambientes de cuidado, pois os profissionais de saúde, neste estudo os enfermeiros, necessitam de qualidade da evidência nos ambientes clínicos baseadas na pesquisa para a tomada de decisão no ponto de cuidado. Os dispositivos tipo PDA promovem suporte necessário para o alcance das competências exigidas no sistema de intensa informação de cuidado. ${ }^{6,36-39}$ Entretanto, tem sido um desafio contínuo aos desenvolvedores e usuários de sis- temas otimizar o fluxo de trabalho, diminuir a sobrecarga de trabalho dos profissionais e adequar o design dos dispositivos tecnológicos e sistemas à realidade da prática.

A categoria Conteúdo do critério Ergonomia obteve média geral 4,86 considerado E pelos avaliadores. Dois itens foram avaliados: informações claras, objetivas e atualizadas (média 4,82) e; o conteúdo está inter-relacionado e consistente com a área da terapia intensiva (média 4,91).

Ao se adotar a CIPE $^{\circledR}$ versão 1.0 como referencial teórico neste estudo, um sistema de classificação mundial para Enfermagem ainda pouco utilizado no Brasil, percebeu-se que esta terminologia fornece uma estrutura de dados, informações e conhecimento que atende as necessidades da prática do enfermeiro, pois integra diagnósticos, intervenções e resultados em cada avaliação clínica do cliente. ${ }^{9} \mathrm{O}$ u seja, continuamente o enfermeiro é estimulado a olhar a evidência da prática, associar com os dados do sistema e tomar a decisão sobre o melhor cuidado a ser prestado. ${ }^{40}$

Ao se utilizar uma terminologia unificada para delinear os elementos constituintes da prática de enfermagem, os enfermeiros poderão comparar suas atividades em diversos contextos clínicos, populacionais, geográficos ou temporais, e também identificarão as suas particulares e fundamentais contribuições frente à equipe multidisciplinar da saúde através da diferenciação e qualidade dos cuidados prestados. ${ }^{9}$ Desta forma, ousa-se afirmar a partir deste estudo que a utilização da $\mathrm{CIPE}^{\circledR} \mathrm{em}$ um dispositivo móvel para o desenvolvimento do PE na UTI melhora a comunicação entre os profissionais de saúde para o cuidado seguro e eficiente ao cliente e reduz os erros associados.

Este sistema informatizado conta, atualmente, com aproximadamente 975 diagnósticos e mais de 825 intervenções de enfermagem elaborados a partir da $\mathrm{CIPE}^{\circledR}$ versão 1.0. Salienta-se que o sistema desenvolvido e avaliado até o momento, possui a capacidade de ampliar seu conteúdo à medida que os enfermeiros têm espaço específico no ambiente para registrar em linguagem livre, novos diagnósticos e intervenções que, porventura, ainda não tenham sido contemplados.

Acredita-se que, a partir do conteúdo desenvolvido, o sistema informatizado do PE poderá oportunizar aos enfermeiros o conhecimento e a condição necessários e adequados para que ele se sinta seguro frente ao processo de tomada de decisão durante a assistência de enfermagem em terapia intensiva. ${ }^{14,17}$ 
Os relatos dos avaliadores reforçam os resultados positivos referente à avaliação do Conteúdo do PE informatizado no dispositivo móvel.

Praticidade, objetividade, modernidade, completo e com possibilidade de acrescentar observações novas se for necessário (E1).

[...] de acordo com diagnóstico já tem as intervenções de enfermagem; tem como alterar qualquer intervenção ou acrescentar (E4).

Disponibilidade de variedade ampla de diagnósticos e intervenções que contemplam os vários sistemas humanos (E5).

O Processo utilizado refere-se à Classificação Internacional das Práticas de Enfermagem, que estabelece uma linguagem comum para descrever os fenômenos dos clientes, as intervenções específicas de enfermagem e os resultados. A construção de sistemas que possam organizar, arquivar e recuperar os dados da prática de enfermagem, a partir de linguagem padronizada, é de suma importância por possibilitar métodos de comparação de práticas [benchmarking] e Guidelines de Enfermagem para apoiar as decisões clinicas e gerar mudanças de padrões e condutas, estudos de avaliação e medidas de resultados em enfermagem. Parabéns pelo trabalho! (Pf1).

Percebeu-se, durante o período da coleta de dados e mediante o número de Diagnósticos e Intervenções validados, que os enfermeiros não apresentaram dificuldades na utilização da CIPE ${ }^{\circledR}$. O raciocínio clínico desenvolvido pelos enfermeiros acompanhou a estrutura de dados do sistema facilitando a aplicação prática, entretanto novos estudos necessitam ser desenvolvidos no sentido de avaliar o impacto deste sistema na prática de enfermagem em terapia intensiva e na segurança do cliente.

Evidencia-se nos relatos dos avaliadores a afirmação da reflexão acima, bem como a facilidade para determinação dos Diagnósticos e Intervenções de Enfermagem: o processo de enfermagem utilizado representa uma forma de sistematização da assistência de enfermagem adequada ao atendimento em terapia intensiva mesmo porque, foi construída para tal. Possibilita avaliação do cliente de forma objetiva, clara, sem repetições desnecessárias. Nos apresenta grande variedade de intervenções de enfermagem atualizadas, oportunizando uma assistência de enfermagem com qualidade (E5).

Excelente, articula todas as etapas [principais] do método científico de resolução de problemas, levantamento de dados, avaliações dos dados, hipóteses de soluções e verificação dos resultados (E9).
A forma com que os dados foram estruturados, ou seja, avaliação, diagnósticos e intervenções, vão ao encontro da lógica de um sistema de informação, onde o dado selecionado, a partir da situação clínica do cliente, gera uma estrutura informacional e, sequencialmente, promove o conhecimento, uma vez que estabelece uma associação de idéias para a tomada de decisão, ou seja, as intervenções de enfermagem necessárias a cada cliente especificamente. Esta associação/ relação estabelecida pelos passos do PE informatizado proporciona aos enfermeiros a ação por meio da intervenção, evidenciando assim a lógica do conhecimento adquirido durante a sua implementação. ${ }^{17}$

A categoria Técnico do critério Ergonomia do sistema obteve média geral 4,46, considerado MB pelos avaliadores. Três itens foram avaliados: estrutura dos dados - como os dados no sistema foram organizados - permite raciocínio compatível com a prática (média 4,45 ); segurança e privacidade das informações (média 4,54) e funcionamento adequado do sistema (média 4,31).

No item, funcionamento adequado do sistema, durante a coleta de dados observou-se necessidade de corrigir queda da conexão entre o ambiente móvel (PDA) e o ambiente via Web; necessidade de ajustar os dados para visualização também em celular e comunicar aos enfermeiros que os dados registrados eram armazenados no ambiente via Web e não no dispositivo móvel. Ou seja, após o registro dos dados no dispositivo móvel os enfermeiros podem acessá-los tanto do ambiente via web quanto do dispositivo móvel.

Quanto ao item segurança e privacidade das informações, utilizou-se um cuidado criterioso, uma vez que este item está diretamente relacionado com as questões éticas da informação que permeia toda a assistência de enfermagem nos mais diversos cenários da prática profissional. Ou seja, para garantir esta propriedade do sistema, foi criado um login e uma senha para cada avaliador, de caráter pessoal e intransferível, que permitia somente a ele o acesso aos dados para avaliação do cliente desejado no momento da implementação do PE informatizado.

Além disso, uma vez que os dados são salvos no ambiente PDA, estes não podem ser alterados, necessitando que o enfermeiro proceda a uma nova avaliação do cliente. Os dados podem ser visualizados pelos profissionais de saúde no ambiente relatório do sistema via Web. Outro ponto que pode ser destacado em relação à segurança 
do sistema é o de que ele não aceita dados inexistentes, como por exemplo, um cliente do sexo masculino com dados de avaliação de uma pessoa do sexo feminino.

Um dos avaliadores sugeriu outras formas de segurança que podem ser adotadas neste sistema informatizado posteriormente, conforme o seguinte relato: A segurança do sistema foi avaliada considerando o acesso restrito por meio de senhas de acesso. Entretanto, não foi possível avaliar outros padrões de segurança como biometria, assinatura digital, acesso a diferentes visões do sistema etc. Ferramentas que talvez possam já ter sido pensadas para o sistema (Pf1).

A Segurança da Informação é compreendida como um conjunto de medidas que visam proteger e preservar informações e sistemas de informações, assegurando-lhes confidencialidade, integridade, disponibilidade, não repúdio e autenticidade. Esses elementos constituem os cinco pilares da segurança da informação e, portanto, são essenciais para assegurar a integridade e confiabilidade em sistemas de informações. ${ }^{41}$

\section{Avaliação do critério usabilidade}

A seguir, é apresentado na Tabela 1, o resultado das avaliações referentes aos itens do critério de Usabilidade do sistema de informação. A Usabilidade visa desenvolver conhecimentos sobre as capacidades, limites e outras características do desempenho humano e que se relacionam com o projeto de interfaces, entre indivíduos e outros componentes do sistema. ${ }^{19}$

Tabela 1 - Avaliação de usabilidade do processo de enfermagem informatizado no Personal Digital Assistant

\begin{tabular}{lc}
\hline Itens de avaliação - usabilidade & Média \\
\hline O programa roda facilmente na plataforma, sem interferências & 3,92 \\
As telas do sistema são claras, fáceis de ler e interpretar & 4,08 \\
O usuário é capaz de acessar o sistema/programa facilmente & 4,77 \\
O menu é viável e fácil de usar (a estrutura da informação disponibilizada é viável e fácil de usar) & 4,54 \\
O menu atende as funções definidas para os objetivos do sistema & 4,62 \\
O programa favorece um tratamento eficiente aos problemas de sua dimensão na UTI & 4,73 \\
É eficiente para o intercâmbio de informações entre o usuário e o programa & 4,77 \\
O programa possui um limite de crescimento apropriado às exigências de uso & 4,67 \\
O programa permite o manejo eficiente dos dados que utiliza & 4,62 \\
As exigências de memória não impedem o programa de rodar & 4,77 \\
O sistema operacional exigido está disponível ou pode ser obtido & 4,85 \\
O sistema possui segurança de dados & 4,77 \\
O sistema não aceita dados inexistentes & 4,62 \\
A conexão e a comunicação entre o módulo fixo e móvel é adequada & 4,54 \\
As exigências de hardware são compatíveis com a realidade & 4,75 \\
O sistema está integrado a um Banco de Dados & 4,92 \\
É fácil adaptar a outros ambientes & 4,83 \\
É fácil instalar em outros ambientes & 4,83 \\
Está de acordo com padrões de portabilidade & 4,69 \\
\hline Média geral & 4,65 \\
\hline
\end{tabular}

$\mathrm{Na}$ avaliação de Usabilidade, a média geral atribuída pelos avaliadores foi 4,65 demonstrando que os avaliadores consideram a usabilidade do sistema informatizado no ambiente móvel tipo PDA como E.

Ao analisar separadamente cada um dos 19 itens avaliados na Usabilidade do PE informatizado, verifica-se que somente os itens: o programa roda facilmente na plataforma, sem interferências (média $=3,92$ ) e as telas do sistema são claras, fáceis de ler e interpretar (média 4,08) foram caracterizados como MB. Destaca-se que todos os outros 17 itens da Usabilidade do sistema foram considerados EXCELENTES pelos avaliadores.

Desta forma, diante dos resultados obtidos das análises e das reflexões realizadas é possível afirmar que o sistema informatizado do PE em UTI de acordo com a CIPE ${ }^{\circledR}$ versão 1.0 desenvolvido e implementado até o momento, possui os critérios de Ergonomia (organização, interface, conteúdo 
e técnico) e Usabilidade, uma vez que foram considerados pelos avaliadores como EXCELENTES. Neste sentido, considera-se que a primeira parte do ciclo desta produção tecnológica se finaliza, ou seja, pode-se concluir que os sistemas do PE fixo (Web) ${ }^{17}$ e móvel (PDA) ${ }^{14}$ desenvolvidos, implementados e diversas vezes avaliados pelos diferentes profissionais é adequado para ser utilizado e implementado na UTI.

\section{CONSIDERAÇÕES FINAIS}

A complexidade das informações no mundo globalizado está redefinindo e criando novas características aos cuidados de saúde, constituindo-se em um atual desafio para a saúde e para a enfermagem. A partir deste estudo, é possível afirmar que o PE em dispositivo tipo PDA a partir da CIPE $^{\circledR} 1.0$ pode ser considerado uma fonte de informações e conhecimentos que proporcionará aos enfermeiros novas modalidades de aprender enfermagem em terapia intensiva, uma vez que é um espaço permanente de reflexões que incentiva também a pesquisa, o diálogo entre os pares e a equipe interdisciplinar, a tomada de decisão e, sobretudo, a melhoria da segurança da prática de enfermagem em UTI.

Não há dúvida de que os enfermeiros desempenham um papel chave em promover e coordenar o cuidado do cliente em terapia intensiva e que estudos que se preocupam com o desempenho deste papel são fundamentais na segurança do cuidado de enfermagem. Neste sentido, este sistema desenvolvido tem uma aplicação prática porque permite avaliar, intervir e gerenciar o cuidado de enfermagem uma vez que proporciona maior segurança e envolvimento dos enfermeiros com as necessidades do cliente à beira do leito.

A CIPE ${ }^{\circledR}$ versão 1.0 que serviu de referência ao estudo é um sistema de classificação padronizado, uma vez que possui uma estrutura adequada para informatizar o PE. A utilização deste sistema de classificação mundial possibilitou aos enfermeiros a organização e o desenvolvimento do raciocínio lógico no processo de assistir/cuidar dos clientes internados em terapia intensiva, pois permitiu a estes estabelecer uma relação concreta entre as avaliações clínicas, os diagnósticos, as intervenções e os resultados de enfermagem através das ferramentas informatizadas.

Apesar da pequena amostra que o representa, não permitindo sua generalização, este estudo é inovador tanto por integrar a pesquisa em en- fermagem com a produção tecnológica quanto por ser o único sistema construído em todo o território nacional utilizando a $\mathrm{CIPE}^{\circledR}$ versão 1.0 para a UTI, e ainda estimula o desenvolvimento de novas pesquisas no sentido de avaliar o impacto deste tipo de tecnologia no desempenho do trabalho do enfermeiro, bem como na melhoria da segurança do paciente.

\section{REFERENNCIAS}

1. Martins CR, Dal Sasso GTM. Tecnologia: definições e reflexões para a prática em saúde e enfermagem. Texto Contexto Enferm. 2008 Jan-Mar; 17(1):11-2.

2. Zuzelo PR, Gettis C, Hansell AW, Thomas L. Describing the influence of technologies on registered nurses' work. Clin Nurse Spec. 2008 MayJun; 22(3):132-40.

3. Hakes B, Whittington J. Assessing the impact of an Electronic medical record on nurse documentation time. Comput Inform Nurs. 2008 Jul-Aug; 26(4):234-41.

4. Kring DL. Clinical nurse specialist practice domains and evidence-based practice competencies a matrix of influence. Clin Nurse Spec. 2008 Jul-Aug; 22(4):179-83.

5. Rosenbloom ST, Grande J, Geissbuhler A, Miller RA. Experience in implementing inpatient clinical note capture via a provider order entry system. J Am Med Inform Assoc. 2004 Jul-Aug; 11(4):310-6.

6. Murphy CA, Merriman K, Zabka C, Penick M, Villamayor P. Patient-entered electronic healthcare records with electronic medical record integration: lessons learned from the field (Paper Presentation). Comput Inform Nurs. 2008 Sep-Oct; 26(5):302.

7. Hannah KJ, Ball M, Edwards MJA. Introduction to nursing informatics. Health Informatics Series. New York (US): Springer; 2006.

8. Organización Panamericana de la Salud - OPAS. Desarrollo de sistemas normalizados de información de enfermería. Washington (US): OPS; 2001.

9. International Council of Nurses - ICN. Classificação Internacional para a Prática de Enfermagem - CIPE ${ }^{\circledR}$ versão 1.0. Tradução: Marin HF. São Paulo (SP): Algol Editora; 2007.

10. International Standards Organization - ISO 18104. Health informatics - Integration of a reference terminology model for nursing. 2003 [acesso 2008 Abr 22]. Disponível em http://www.iso.org/iso/ iso_catalogue/catalogue_tc/catalogue_detail. htm?csnumber $=33309$

11. Driscoll WD, Columbia MA, Peterfreund RA. An observational study of anesthesia record completeness using an anesthesia information management system. Anesth Analog. 2007 Jun; 104:1454-61. 
12. Kossman SP. Perceptions of impact of electronic health records on nurses' work. Stud Health Technol Inform. 2006; 122:337-41.

13. Brandt K. Poor quality or poor design? A review of the literature on the quality of documentation within the electronic medical record (Paper Presentation). Comput Inform Nurs. 2008 Sep-Oct; 26(5):302-3.

14. Barra DCC. Processo de enfermagem informatizado em terapia intensiva em ambiente PDA (Personal Digital Assistant) a partir da $\mathrm{CIPE}^{\circledR}$ versão 1.0 [dissertação]. Florianópolis (SC): Universidade Federal de Santa Catarina. Programa de PósGraduação em Enfermagem; 2008.

15. Sasso GTMD. Uma proposta do processo de enfermagem informatizado em terapia intensiva a partir da CIPE versão $\beta 1$. São José (SC): Instituto de Cardiologia - Secretaria de Estado da Saúde de Santa Catarina; 1999.

16. Zabotti C, Souza J. Metodologia eletrônica de cuidados de enfermagem aos pacientes em terapia intensiva com alterações respiratórias utilizando a CIPE [trabalho de conclusão de curso]. Palhoça (SC): Universidade do Sul de Santa Catarina. Faculdade de Enfermagem; 2002.

17. Antunes CR. Processo de enfermagem informatizado ao paciente politraumatizado de terapia intensiva via web [dissertação]. Florianópolis (SC): Universidade Federal de Santa Catarina. Programa de PósGraduação em Enfermagem; 2006.

18. Polit DF, Beck C, Hungler BP. Fundamentos de pesquisa em enfermagem: métodos, avaliação e utilização. Porto Alegre (RS): Artmed; 2004.

19. International Standars Organization/International Electrotechnical Commission ISO/IEC 9241-11 Usability Net. 2006 [acesso 2007 Jul 17]. Disponível em: http://www.usabilitynet.org/tools/r_ international.htm

20. Ministério da Saúde (BR), Conselho Nacional de Saúde, Comissão Nacional de Ética em Pesquisa. Resolução $N^{\circ} 196$ de 10 de outubro de 1996: diretrizes e normas regulamentadoras de pesquisa envolvendo seres humanos. Brasília (DF): MS; 1996. [acesso 2007 Mai 26]. Disponível em: http://www. bioetica.ufrgs.br/res19696.htm

21. Figueiredo RM, Zem-Mascarenhas SH, Napoleão AA, Camargo AB. Caracterização da produção do conhecimento sobre sistematização da assistência de enfermagem no Brasil. Rev Esc Enferm USP. 2006; 40(2):299-303.

22. Marin HF, Cunha ICKO. Perspectivas atuais da informática em enfermagem. Rev Bras Enferm. 2006 Maio-Jun. 59(3):354-7.

23. Évora YDM. A enfermagem na era da informática. Rev Eletr Enferm [online]. 2007 [acesso 2008 Mar 20]; 9(1):14: Disponível em: http://www.fen.ufg. br/revista/v9/n1/v9n1a01 pt.htm
24. Cardoso JP, Rosa VA, Lopes CRS, Vilela ABA, Santana AS, Silva ST. Construção de uma práxis educativa em informática na saúde para ensino de graduação. Ciênc Saúde Coletiva. 2008 Jan-Fev. 13(1):283-8.

25. Fonseca CMBM, Santos ML. Tecnologias da informação e cuidado hospitalar: reflexões sobre o sentido do trabalho. Ciênc Saúde Coletiva. 2007 Maio-Jun. 12(3):699-708.

26. Stricklin ML, Bierer SB, Struk C. Home care nurses' attitudes toward computers. A confirmatory factor analysis of the stronge and brodt instrument. Comput Inform Nurs. 2003 Mar-Apr; 21(2):103-1.

27. Dillon TW, Blankenship R, Crews TJr. Nursing attitudes and images of electronic patient record systems. Comput Inform Nurs. 2005 May-Jun; 23(3):139-45.

28. Jerant AF, Hill DB. Does the use of electronic medical records improve surrogate patient outcomes in outpatient settings? J Fam Pract. 2000 Apr; 49(4):349-57.

29. Moody LE, Slocumb E, Berg B, Jackson D. Electronic health records documentation in nursing: nurses' perceptions, attitudes, and preferences. Comput Inform Nurs. 2004 Nov-Dec; 22(6):337-44.

30. Smith K, Smith V, Krugman M, Oman K. Evaluating the impact of computerized clinical documentation. Comput Inform Nurs. 2005 May-June; 23(3):132-8.

31. Scott JT, Rundall TG, Vogt TM, Hsu J. Kaiser permanente's experience of implementing an electronic medical record: a qualitative study. BMJ. 2005 Dec; 331(7528):1313-6.

32. Rose AF, Schnipper JL, Park ER, Poon EG, Li Q, Middleton B. Using qualitative studies to improve the usability of an EMR. J Biomed Inform. 2005 Feb; 38:51-60.

33. Poissant L, Pereira J, Tamblyn R, Kawasumi Y. The impact of electronic health records on time efficiency of physicians and nurses: a systematic review. J Am Med Inform Assoc. 2005 Sep-Oct; 12(5):505-16.

34. Ammenwerth E, Kutscha A, Eichstadter R, Haux R. Systematic evaluation of computer-based nursing documentation. Medinfo. 2001; 10(Pt 2):1101-6.

35. Kaplan B. Evaluating informatics applications-some alternative approaches: theory, social interactionism, and call for methodological pluralism. Int J Med Inform. 2001 Nov; 64(1):39-56.

36. Whitten P, Johannessen LK, Soerensen T, Gammon D, Mackert M. A systematic review of research methodology in telemedicine studies. East Lansing (US): Michigan State University; 2007 May 15.

37. Kendrick DC, Bu D, Pan E, Middleton B. Crossing the evidence chasm: building evidence bridges from process changes to clinical outcomes. J Am Med Inform Assoc 2007 May-Jun; 14: 329-39. 
38. Rempher KJ, Lasome CE, Lasome TJ. Leveraging palm technology in the advanced practice nursing environment. AACN Clin Issues. 2003 Aug; 14: 363-70.

39. Sloan H L, Delahoussaye CP. Clinical application of the Omaha System with the Nightingale Tracker: a community health nursing student home visit program. Nurse Educ. 2003 Jan-Feb; 28:15-7.

40. Rothwell K. Devices \& technology. In: Advances in technology revolutionizing patient care. Nursezone
Web site. [acesso 2008 Nov 21]. Disponível em: http://www.nursezone.com/include/PrintArticle. asp? articleid $=7488$.

41. Silva Filho AM. Segurança da informação: sobre a necessidade de proteção de sistemas de informações. Rev Espaço Acadêmico [online]. 2004 Nov [acesso 2008 Jun 15]; 42(IV). Disponível em: http:/ /www. espacoacademico.com.br/042/42amsf.htm
Correspondência: Daniela Couto Carvalho Barra

Rua Maestro Aldo Krieger, 108, ap. 804

88037-500 - Córrego Grande, Florianópolis, SC, Brasil

E-mail: danyccbarra@yahoo.com.br
Recebido em: 5 de dezembro de 2008 Aprovação final: 23 de novembro de 2009 